\title{
Clinical characteristics and visual outcomes in infectious scleritis: a review
}

This article was published in the following Dove Press journal:

Clinical Ophthalmology

4 November 2013

Number of times this article has been viewed

\author{
Emeline Radhika Ramenaden \\ Veena Rao Raiji \\ Department of Ophthalmology, \\ George Washington University, \\ Washington, DC, USA
}

\begin{abstract}
Infection is a very important but rare cause of scleritis, occurring in about $5 \%-10 \%$ of all patients presenting with scleral inflammation. However, due to the similarity of its presentation, infectious scleritis is often initially managed as autoimmune, potentially further worsening its outcome. The overall visual outcome in infectious scleritis is generally worse than its autoimmune counterparts, perhaps because of the delay in diagnosis or because of the aggressive nature of associated microbes. Thus, there is a definite need for insight into the diagnostic approach and treatment options for this ocular disease process. Several studies and case reports have been published in recent years that have provided useful information regarding the presenting clinical features and etiologic microbial agents in infectious scleritis. This review summarizes the important findings in the literature that may aid in differentiating infectious scleritis from other etiologies, including predisposing factors, microbe-specific characteristics, diagnostic tools, treatment modalities, and outcomes.
\end{abstract}

Keywords: infectious scleritis, Pseudomonas, necrotizing scleritis, abscess

\section{Background}

Scleritis is a state of ocular inflammation with a wide spectrum of clinical presentations and etiologic factors (Table 1). Specific etiologies of scleritis, varying from idiopathic to autoimmune to infectious, portend variable disease severity and outcome. Infection is an important but rare cause of the scleritis, occurring in about $5 \%-10 \%$ of all cases. ${ }^{1}$ However, due to the similarity of its presentation, infectious scleritis is often initially managed as autoimmune, potentially worsening its outcome. The overall visual outcome in infectious scleritis is generally worse than its autoimmune counterparts, perhaps because of this delay in diagnosis or because of the aggressive nature of associated microbes. Recent studies have identified specific inciting factors for infectious scleritis. Not surprisingly, these factors are typically surgery, most commonly pterygium surgery but also excisions of conjunctival neoplasms, cataract surgery, vitreoretinal surgeries, and glaucoma surgeries. ${ }^{2-4}$ Paula et al and Hodson et al report that the use of concomitant radiation or mitomycin $\mathrm{C}$ is also a risk factor. ${ }^{2,5}$ Trauma, especially with introduction of organic material to the ocular surface or self-inoculation from a distant site on the body is also a significant inciting factor. ${ }^{6}$ In isolated cases, immunosuppression due to human immunodeficiency virus or chemotherapy, may be a risk factor for spontaneous cases of infectious scleritis. , $^{2,8}$ Interestingly, Meyer et al reported an individual case of spontaneous infectious scleritis without any such prior history that revealed Pseudomonas aeruginosa resistant to 4th generation fluoroquinolones, indicating the importance of considering infectious etiologies despite the lack of leading history. ${ }^{9}$

\footnotetext{
Correspondence: Emeline Radhika

Ramenaden

Department of Ophthalmology,

George Washington University,

2150 Pennsylvania Ave, NW, Suite 2A,

Washington, DC 20037, USA

Tel + I 20274 I 2800

Fax + I 202 74I 2805

Email radhikar@gwmail.gwu.edu
}

submit your manuscript | www.dovepress.com

Dovepress

http://dx.doi.org// 0.2147/OPTH.S37809 
Table I Types of scleritis

Anterior-diffuse

Anterior-nodular

Anterior-necrotizing (with inflammation) (without inflammation)

Posterior scleritis

Infectious scleritis
Anterior-necrotizing

Greater than $50 \%$ contiguous scleral involvement. Associated with systemic disease. Generally in women $40-70$ years old or men $30-60$ years old. Rare in the young and the very old.

Distinct nodules present. Associated with isolated disease. Both sexes generally involved, 40-60 years old. Scleral necrosis with scleromalacia. Associated with systemic disease. Generally in women in their 40s. Scleral necrosis without scleromalacia. Usually associated with systemic disease. Generally in women, 35-75 years old. Usually associated with anterior scleritis. Generally in women in their 60s. Diagnosis made using clinical and ultrasound findings. Generally necrotizing. Can have anterior and posterior involvement. Scleral ulcers with calcific plaque at the base and microabscesses. Tissue extension beyond obvious clinical findings.*

Notes: *Though infectious scleritis can present in many ways, these are the unique clinical findings found most commonly in this review. Data from Raiji et al, ${ }^{45}$ and Watson and Hayreh. ${ }^{46}$

While postoperative worsening of scleritis has been well reported in those with preexisting autoimmune scleritis, a concomitant infectious etiology is much less common. The similarities in presentation between autoimmune and infectious scleritis often delays diagnosis. Infectious scleritis after pterygium surgery with adjunctive $\beta$-radiation and mitomycin $C$ has been well established. ${ }^{2,11,12}$ Several reasons may exist for this association. Meallet proposed in 2006 that the use of adjunctive therapies such as these are likely to compromise the integrity of episcleral conjunctival vessels and underlying tissue, thus inhibiting adequate wound healing and leaving the sclera vulnerable to infection. He further hypothesizes that surgical techniques such as leaving bare sclera exposed or excessive use of cautery, as well as poor contact lens hygiene or nonhealing epithelial defects may all contribute. ${ }^{12}$ P. aeruginosa, the most common agent in infectious scleritis, utilizes neutrophil-activated collagenases to destroy tissue, especially when sclera is left bare. Other microbes have similar biochemical mechanisms by which they are able to infiltrate ocular tissues. ${ }^{12}$ Furthermore, traditional antibiotic regimens, both topical and systemic, remain inadequate due to the avascularity of the sclera and the dense structure of collagen fibers, limiting tissue penetration. ${ }^{12}$ Additionally, the misdiagnosis of infectious scleritis as a postoperative inflammatory complication leads to initial aggressive local and systemic corticosteroid therapy that further worsens their infection and may lead to poor visual outcomes.
Another known characteristic of infectious scleritis is its sometimes delayed presentation, occurring months to years after ocular surgery or trauma. Jain et al proposed that conjunctival and tear film alterations may expose underlying necrotic scleral collagen to microorganisms and allow localization, adherence, colonization and invasion. ${ }^{1}$ Microbes can then remain dormant within the sclera for years without inciting an inflammatory response, making the diagnosis of infectious scleritis in these cases quite difficult. It remains unclear why these infections may be reactivated after such long periods of latency. ${ }^{12}$

According to one large retrospective study by Hodson et al, the median age of the patients at diagnosis of infectious scleritis is 70 years old. ${ }^{2}$ Most studies reveal an older population, suggesting that age may hold some significance in the diagnosis of infectious scleritis. ${ }^{2,13,14}$ Additionally, the time from an identifiable inciting event to the presentation of scleritis can range in 0 days up to 36 years, generally longer in postsurgical patients than the post-nonsurgical trauma patients, with the longest latency period reported occurring after a history of pterygium surgery. ${ }^{2,10}$

In all cases of infectious scleritis, $P$. aeruginosa is the most common infectious agent. ${ }^{1,2}$ Additional identified microbes include Nocardia, Aspergillus, Fusarium, Candida, Beauveria, Stenotrophomonas, Streptococcus, Staphylococcus aureus (especially of the methicillin-resistant type), Serratia, Enterobacter, Achromobacter, Propionibacterium, Haemophilus, Alcaligenes, mycobacteria, and herpes viridae. In some cases, coinfections have been reported. ${ }^{2,15}$ Although P. aeruginosa as well as other bacterial and viral etiologies are most common in developed countries, fungi and Nocardia are relatively more prevalent in developing countries, perhaps due to humid climates and increased exposure to soil. ${ }^{1}$

Retinal surgeries such as vitrectomy, scleral buckling with intrascleral fixation, and epiretinal membrane peel procedures have been associated with acid-fast organisms, such as Mycobacterium chelonae, more so than anterior segment procedures. ${ }^{2,16}$ These retinal surgeries have additionally been associated with Pseudomonas, methicillin-resistant Staphylococcus aureus, and coagulase-negative Staphylococcus albus., ${ }^{3,17}$ The use of sub-Tenon's triamcinolone acetonide injection has also been associated with the development of infectious scleritis in two cases, which were culture positive for Staphylococcus epidermidis and Nocardia. ${ }^{18,19}$

\section{Presentation}

Infectious scleritis may present with certain clinical patterns that might facilitate more efficient diagnosis when recognized. 
Furthermore, because the prevalence of certain microbes in infectious scleritis varies by environment, inciting event, or sometimes even by symptomology, knowledge of this information may allow faster microbial identification.

Similar to patients with autoimmune scleritis, patients with infectious scleritis commonly present with symptoms of redness, pain, and epiphora. ${ }^{13}$ Infectious scleritis after retinal surgery tends to be associated with more rapid and extreme pain than anterior segment surgeries. ${ }^{3}$ The most common clinical signs at presentation in a retrospective study of 55 patients with infectious scleritis included episcleral and conjunctival hyperemia (98\%) and scleral necrosis $(93 \%),{ }^{2}$ noted consistently in other studies as well. ${ }^{1,12,13}$ In this large study, 19 eyes had isolated scleritis whereas 37 involved adjacent ocular structures, including the cornea and extraocular muscles, both spreading primarily from the sclera to elsewhere or secondarily to the sclera. ${ }^{2}$ An anterior chamber reaction of greater than $1+$ cell is usually present at the initial encounter. ${ }^{20,21}$ Endophthalmitis has also been documented as the presenting finding. ${ }^{1,11,13,22}$ Not surprisingly, retinal surgeries predisposed patients with infectious scleritis to posterior scleral thickening and retinal or choroidal detachments. $3,16,17,21$

More notably, patients with infectious scleritis may present with a scleral ulcer at sites of prior tissue penetration, at times including satellite abscesses or involving extraocular muscles, which may mimic orbital inflammatory syndrome. ${ }^{1,13,21}$ Calcified plaques are often found at the base of scleral ulcers (Figure 1). ${ }^{1,21}$ Unifocal or multifocal scleral abscesses are consistently reported, appearing as distinctly yellowish nodules under intact conjunctiva (Figure 2). ${ }^{1,13,23}$ Abscesses are characteristically scattered superiorly or inferiorly along an arc of $3-4 \mathrm{~mm}$ from the limbus (Figure 3 ). ${ }^{21}$
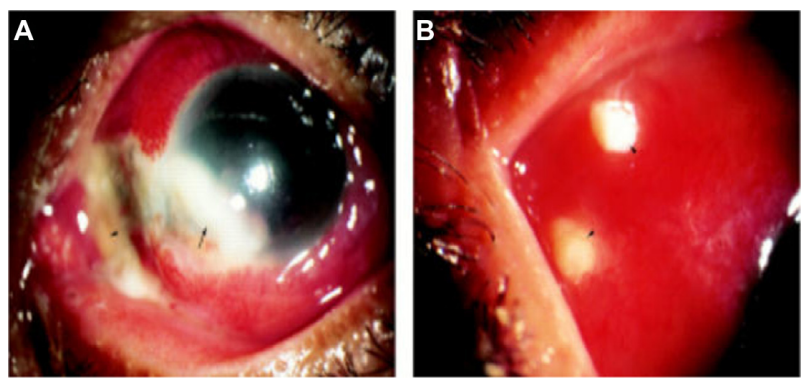

Figure I Scleral ulcer with calcific plaque.

Notes: (A) At presentation, there was a calcific plaque over the scleral ulcer bed (arrowhead). The ulcer progressed and became contiguous with a corneal infiltrate (arrow). (B) Multiple new nodules (arrowheads) from which P. aeruginosa was cultured, appeared on the 22nd day of hospitalization. (CI 998 BMJ Publishing Group. Reproduced with permission from Hsiao CH, Chen J, Huang SC, Ma HK, et al. Intrascleral dissemination of infectious scleritis following pterygium excision. $\mathrm{Br}$ J Ophthalmol. 1998;82(I):29-34. ${ }^{21}$
A black arc-shaped band from the initial ulcer site through subsequent abscesses has been identified in some patients after resolution, indicating a track of disseminated infection. ${ }^{21}$

Necrotizing scleritis tends to be the most common presentation of infectious scleritis. A necrotizing pattern is also seen in association with autoimmune scleritis with or without vasculitis or metabolic disorders, all of which require immunosuppression for treatment and would worsen visual outcome in infectious scleritis (Figure 4). ${ }^{1,15}$ Thus, infectious etiologies should be suspected in any case of progressive indolent necrosis with suppuration, refractory to antiinflammatory regimens. ${ }^{1}$ Mild to moderate pain is generally associated with diffuse and nodular scleritis whereas severe pain is more frequently associated with necrotizing scleritis, ${ }^{24}$
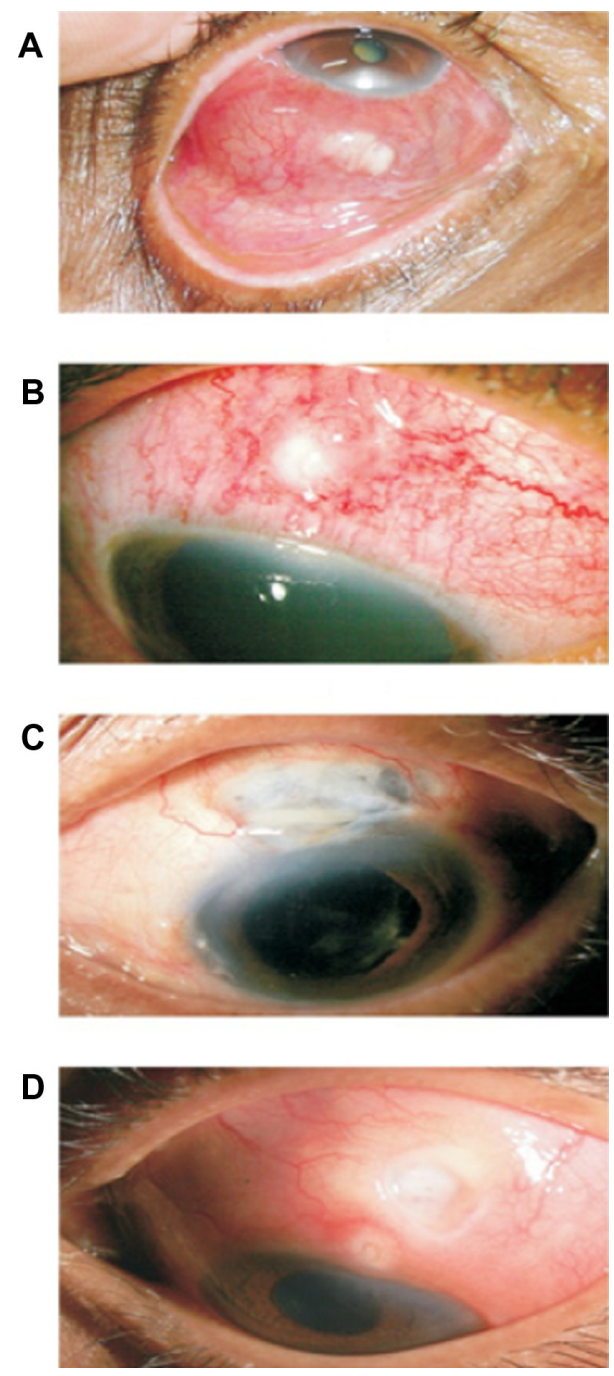

Figure 2 Slit lamp pictures depicting different clinical presentation.

Notes: (A) Case no 2: multiple scleral abscess. (B) Case no 12: single scleral abscess. (C) Case no 4: necrotic ulcer (post cataract surgery). (D) Case no 3: two punched out ulcers. Reproduced with permission from Kumar SS, Das S, Sharma S et al. Clinico-microbiological profile and treatment outcome of infectious scleritis: experience from a tertiary eye care eye center of India. Int $K$ Inflam. 2012:753560.13 


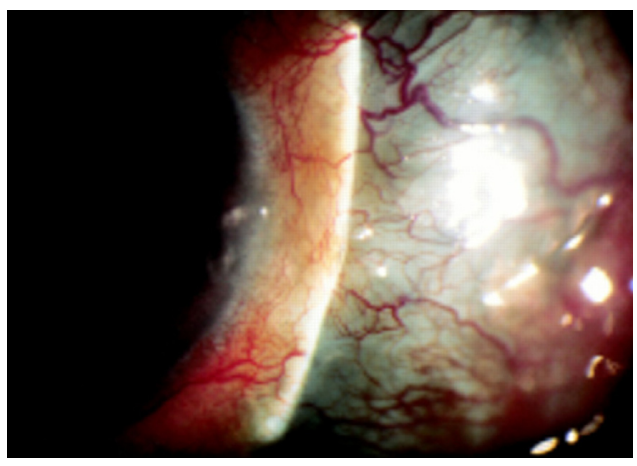

Figure 3 Case I: Scleral thinning from original ulcer through subsequent abscess extended in an arc shape.

Note: () 1998 BMJ Publishing Group. Reproduced with permission from Hsiao CH, Chen JJ, Huang SC et al. Intrascleral dissemination of infectious scleritis following pterygium excision. BrJ Ophthalmol. 1998;82(1):29-34.21

suggesting that pain out of proportion to examination findings may be indicative of a possible infectious etiology.

Other ocular complications noted at the time of presentation of infectious scleritis are decreased vision, peripheral keratitis presenting as interstitial keratitis or thinning with ulceration, or glaucoma. ${ }^{2,20}$

For the purposes of this review, infectious agents that have not been shown in the literature to cause primary scleritis were excluded.

\section{Diagnosis}

In two large retrospective reviews done in the United States and Taiwan, the majority of cases of infectious scleritis were

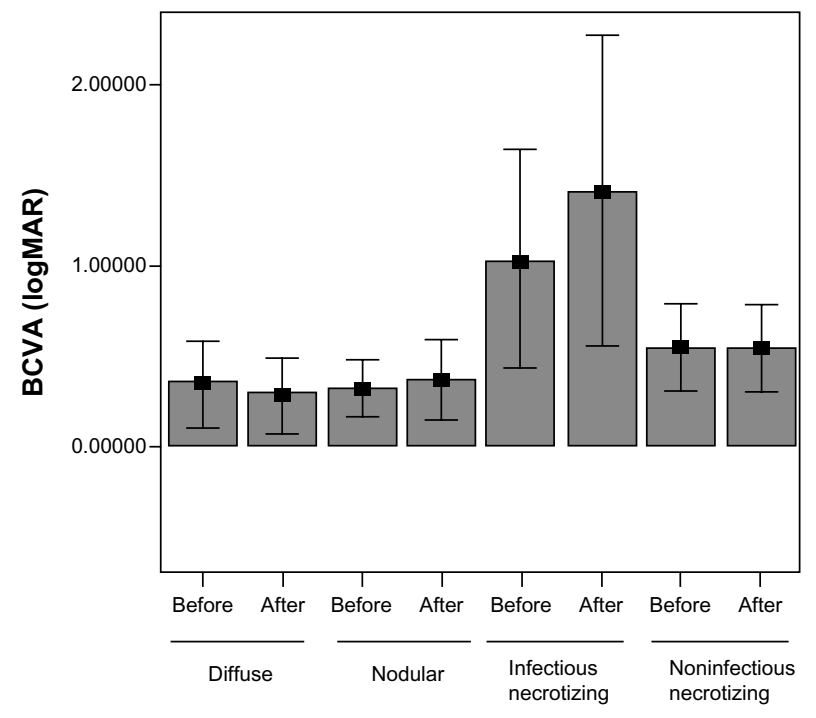

Figure 4 Visual acuities before and after treatment.

Notes: Patients with infectious necrotizing scleritis had the poorest BCVA before and after treatment. The data are presented as the mean \pm standard deviation. Reproduced with permission from Ahn SJ, Oh JY, Kim MK, Lee JH, Wee WR. Clinical features, predisposing factors, and treatment outcomes of scleritis in the Korean population. Korean J Ophthalmol. 2010;24(6):331-335. ${ }^{15}$

Abbreviations: BCVA, best corrected visual acuities; logMAR, logarithmic value of the minimal angle of resolution. found to be bacterial, with fewer cases being fungal. ${ }^{2,22}$ In developing countries, however, fungal etiologies are more common, likely related to differences in climate and environmental exposures. ${ }^{1,13}$

In general, fungal, nocardial and mycobacterial infections take longer to diagnose than bacterial infections. ${ }^{2}$ It is recommended that a complete diagnostic work-up of infectious etiologies include scleral scrapings and culture on blood and chocolate agar, brain-heart infusion broth, thioglycolate broth, nonnutrient agar with $E$. coli overlay, and Sabouraud dextrose agar. ${ }^{1}$ Diagnosis of viral etiologies like herpes-related scleritis can be confirmed by scleral biopsy and the use of immunofluorescence, as well as positive titers or signs of chronic herpetic infection like corneal hypoesthesia. ${ }^{14,20}$ Kumar et al advocate scleral scrapings in every case of suspected infectious scleritis that does not respond to initial antibiotics, not only for the purpose of diagnosis but also to debulk necrotic tissue and improve antimicrobial penetration. ${ }^{13}$

In addition to cues from clinical presentation, history, and slit-lamp examination, the use of ancillary testing has been proposed to be of diagnostic value in infectious scleritis. Nguyen and Yiu comment on the utility of ultrasound biomicroscopy in allowing early detection of scleritis-associated retinal and choroidal detachments. ${ }^{25}$ Ultrasound biomicroscopy is further used to demonstrate anterior ciliary body rotation and elimination of the ciliary sulcus in infectious scleritis along with a diffuse lacy-appearing choroidal thickening. ${ }^{25}$ Additionally, they suggest that optical coherence tomography allows the visualization of small vitreoid opacities and abnormal subretinal deposits, which may represent lipofuscin-laden macrophages in infectious scleritis (Figure 5). ${ }^{25}$

Su et al performed a prospective study in which they found that elevated erythrocyte sedimentation rate and C-reactive protein were noted in 9 of 12 patients with infectious scleritis. ${ }^{10}$ While these general inflammatory parameters can be elevated in any case of inflammation, in these patients, both of these values returned to normal with treatment of the infection. ${ }^{10}$ Additionally, these patients underwent a thorough laboratory work-up to document the lack of any associated underlying autoimmune state that could have explained these laboratory findings.

\section{Microbe-specific findings}

Bacterial etiologies comprise the majority of cases of infectious scleritis. ${ }^{5}$ Bacterial proteins may contribute to tissue damage via proteases and activation of tissue complement pathways. ${ }^{5}$ Pseudomonas aeruginosa represents about $85 \%$ of cases of bacterial scleritis. ${ }^{5}$ Thus, most findings discussed 


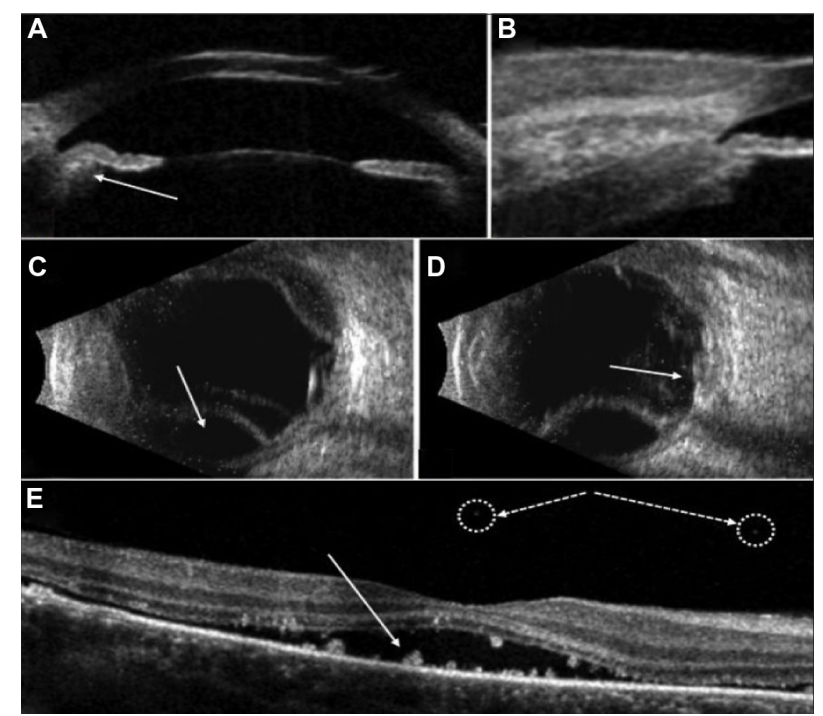

Figure 5 Advanced imaging studies of complications of infectious scleritis.

Notes: (A) $35 \mathrm{MHz}$ immersion ultrasound biomicroscopy demonstrating shallow anterior chamber, thickened and anteriorly rotated ciliary body (arrow), and elimination of the ciliary sulcus. (B) Thickened episcleral, scleral, and choroidal tissues are evident in the magnified view. (C) $10 \mathrm{MHz}$ B-scan ultrasonography showing double retinal and choroidal detachment, L-I2 view, (D) L-mac view and (E) spectral optical coherence tomography showing vitreous clumps (dotted arrows and circles), subretinal fluid, and subretinal precipitates (solid arrow). Reproduced with permission from Nguyen P, Yiu SC. Imaging studies in a case of infectious scleritis after pterygium excision. Middle East Afr J Ophthalmol. 2012;19(3): $337-339 .{ }^{25}$

Abbreviations: L- 12, longitudinal-12 view; L-mac, longitudinal-macular view.

in relevant studies are largely in the context of Pseudomonas scleritis. Though these findings are generally shared by most infectious etiologies, there are certain characteristics or outcomes associated with microbes other than Pseudomonas that may be indicative of other etiologies.

Staphylococcus aureus is also an established cause of bacterial scleritis. As reported by Arora et al, S. aureus scleritis presented as a pyomyositis with an inverse hypopyon and localized scleral thinning in a patient with a large thigh abscess. ${ }^{26}$ Lee et al reported a case of methicillin-resistant S. aureus 6 months after pterygium excision that was initially misdiagnosed clinically as Pseudomonas and was unresponsive to systemic amikacin and ceftazidime as well as topical ciprofloxacin. They suggest that MRSA should be suspected when typical postoperative antibiotics are ineffective. ${ }^{27}$

Streptococcus pneumoniae is a common infectious agent in children and the elderly with concomitant joint, cardiovascular, gastrointestinal, and genitourinary infections. Recently, this bacteria has been associated with infectious scleritis after pterygium surgery with concurrent use of $\beta$-irradiation or mitomycin C. ${ }^{5,11}$ Paula et al reported a case of S. pneumoniae scleritis that presented with mucopurulent discharge, vascular congestion, and three scleral abscesses with necrosis and a small aqueous fistula in the upper sclera at the site of pterygium excision. This case responded well to topical ciprofloxacin and intravenous cephatholin as well as oral diclofenac. ${ }^{5}$ Altman et al discussed four cases of $S$. pneumoniae scleritis that were treated with intravenous and topical antibiotics, two of which had full resolution and good visual outcome and two that had more devastating outcomes. One of these latter patients developed endophthalmitis requiring enucleation and one developed scleral perforation requiring a corneoscleral graft with resulting count fingers visual outcome. ${ }^{11}$ Therefore, $S$. pneumoniae represents a potentially aggressive form of infectious scleritis.

Stenotrophomonas maltophilia is a rare aerobic nonfermentative Gram-negative bacterium that is difficult to treat. Lin et al reported a case of S. maltophilia-associated infectious scleritis that occurred 18 years after pterygium surgery. ${ }^{28}$ Ramos-Esteban and Jeng reported a case of S. maltophilia scleritis in which ultrasound biomicroscopy revealed a dome-shaped mass over an area of a partial-thickness scleral laceration after minor ocular trauma. ${ }^{29}$ In one particular case, full resolution seemingly occurred after fortified topical and systemic antibiotics as well as surgical debridement and patch grafting. Interestingly, however, 5 months after resolution, this patient presented with intrascleral dissemination of the same infection, indicating the importance of long-term follow-up and the possibility of microinvasion greater than clinically detectable..$^{28}$

Mycobacterium tuberculosis scleritis is rare. The mycobacteria itself can cause an immune-mediated inflammatory microangiopathy and may indirectly lead to an inflammatory scleritis. ${ }^{1}$ Kesen et al reported a case of a 54-year-old woman with 6 months of eye pain and nodular scleritis unresponsive to steroids and methotrexate. She was found to be QuantiFERON ${ }^{\circledR}$ gold-positive with hilar granulomas on $\mathrm{CT}$, but due to initial lack of response to quadruple tuberculosis therapy and a bronchoalveolar lavage negative for acid fast bacilli, the regimen was abruptly discontinued and immunosuppression was restarted. She later presented with scleral rupture and polymerase chain reaction of the specimen at that time revealed the M. tuberculosis genome. ${ }^{30}$ Mycobacterial infections other than tuberculosis (MOTT) have a low incidence in humans but present in a disseminated form in immunocompromised patients. These microbes are known to be difficult to isolate by common laboratory techniques and are easily confused with other pathogens. Therefore, they should be suspected in immunocompromised patients with +AFB (acid-fast bacilli) or granulomatous noncaseating inflammation. ${ }^{31} \mathrm{M}$. chelonae is among these MOTT species and has been shown to cause scleritis. ${ }^{31} M$. 
chelonae is a quick-growth mycobacteria easily confused with Nocardia or M. fortuitum that requires several tissue samples to adequately differentiate, thereby delaying diagnosis and treatment. It is known to be resistant to typical antituberculous drugs. Treatment must be continued for 4 weeks to 6 months after full resolution of clinical signs, although due to the rarity of this diagnosis, the exact time of treatment has not yet been established. ${ }^{31}$

Lepromatous leprosy, another mycobacterium-related disease, has been reported to present with unilateral ocular involvement as interstitial keratitis and granulomatous anterior uveitis. In one report by Poon et al, an initial response was achieved using antileprotics and anti-inflammatory agents; however, the patient went on to develop recurrent episodes of scleritis that necessitated multiple scleral patch grafts for scleral thinning, subsequently requiring enucleation. Histology failed to demonstrate lepromatous infection, but showed chronic nongranulomatous scleritis. ${ }^{32}$

Toxoplasma gondii, an obligate intracellular parasitic protozoan, is usually associated with posterior intraocular inflammation; however, it has been reported by Schuman et al to cause scleritis in five cases. ${ }^{33}$ Two of those patients were diagnosed clinically and treated successfully with medical therapy. Three of the patients, two of whom were immunosuppressed, went undiagnosed without treatment for toxoplasmosis, and progressed to eventually require enucleation. Thus, T. gondii, while rare, should be suspected in scleritis with associated retinochoroiditis, especially in those who are immunosuppressed. ${ }^{33}$

Although herpes infections of the eye, both HSV and VZV, have predominantly been thought of in relation to the cornea and ocular adnexa, they have also been reported in cases of infectious scleritis. ${ }^{14}$ Gonzalez-Gonzalez et al conducted a large retrospective study of patients with herpetic scleritis and found a predominance of middle-aged females with unilateral findings and moderate to severe pain. ${ }^{20}$ The acuity of the presentation of herpes scleritis can be variable. While one study reports acute onset, ${ }^{20}$ another reports chronic herpes-associated inflammation with an average of 3.2 years of symptoms prior to diagnosis. ${ }^{14}$ Herpetic scleritis is more likely to be of the diffuse anterior type than of the nodular or necrotizing type. ${ }^{20}$ Perilimbal devascularization with peripheral corneal thinning along with a significant amount of associated uveitis are findings associated with herpetic scleritis, with significantly greater vision loss noted in herpes than in idiopathic cases of scleritis. ${ }^{14}$ In general, herpes infections are not associated with posterior scleritis, vitreoretinal involvement, or scleromalacia perforans. ${ }^{14,20}$
Herpetic scleritis has similar rates of increased intraocular pressure when compared to idiopathic types. ${ }^{20}$ Interestingly, systemic symptoms are generally not present in patients with herpetic scleritis, and the central cornea tends to be spared. ${ }^{14}$ This lack of typical corneal findings in herpetic scleritis generally delays its diagnosis; therefore, a high index of suspicion should exist when the previously discussed symptoms and signs are noted. ${ }^{20}$ Definitive diagnosis can be made by immunohistopathological analysis of scleral biopsies, clinical evidence of dendritic or stromal keratitis, or positive anti-HSV or anti-VZV titers. ${ }^{20}$ Herpetic scleritis typically responds quickly to acyclovir treatment, within 3-8 weeks, ${ }^{14,20}$ with the inflammation generally lasting from 5-32 months. ${ }^{14}$ The initial dosage of acyclovir used in these studies was $800 \mathrm{mg}$ five times daily, ${ }^{14,20}$ with most patients requiring a lower maintenance dose of acyclovir to prevent recurrence, which typically occurs at the same site as previous inflammation. ${ }^{14}$ Previous treatment with immunosuppressive therapy can prolong and worsen herpetic scleral inflammation. ${ }^{14}$

Fungal scleritis, unlike bacterial and viral scleritis, has worse overall outcomes (Table 2). ${ }^{1,34}$ Jain et al report that fungal scleritis is more commonly encountered in hot, humid climates, generally following surgical or accidental trauma. ${ }^{1}$ In cases of fungal scleritis, full thickness corneal inflammation contiguous with scleral lesions is quite common. ${ }^{1}$ In their study, patients with fungal scleritis progressed despite treatment, to develop rapidly progressive cataracts, serous retinal or choroidal detachments, phthisis, or endophthalmitis, of which a majority progressed to evisceration. ${ }^{1}$

Jain et al further report that Aspergillus and Nocardia are the most common fungal agents in infectious scleritis. ${ }^{1}$ Fincher and Fulcher recovered Aspergillus fungal hyphae from a biopsy of a scleral nodule in one patient with a known history of intravenous drug abuse. ${ }^{35}$ This infection was successfully treated with intravenous caspofungin as

Table 2 Comparison of fungal cases with other infectious scleritis cases

\begin{tabular}{lll}
\hline & Fungal scleritis & Others \\
\hline Total number & 8 & 13 \\
Associated corneal & $3 / 8(40 \%)$ & $4 / 13(30 \%)$ \\
infiltration & & \\
Multifocal scleral abscess & $3 / 8(40 \%)$ & 0 \\
Endophthalmitis & $3 / 8(40 \%)$ & 0 \\
Eviscerated & $4 / 8(50 \%)$ & 0 \\
Useful vision & $1 / 8(13 \%)$ & $6 / 13(46 \%)$ \\
& $(95 \% \mathrm{Cl}, 0 \%-36 \%)$ & $(95 \% \mathrm{Cl}, 19 \%-63 \%)$ \\
\hline
\end{tabular}

Note: Microbial scleritis - experience from a developing country. () 2008 Nature Publishing Group. Reproduced with permission from: Jain V, Garg P, Sharma S. Microbial scleritis-experience from a developing country. Eye (Lond). 2009;23(2):255-261.' 
well as repeated surgical drainage of all emerging scleral abscesses. ${ }^{35}$ Nocardia is much more common in agricultural societies, where exposure to soil or plant matter is prevalent, as compared with other infectious etiologies. ${ }^{1,34}$ Generally, Nocardia does not cause fulminant infection; however, in the presence of immunosuppression such as oral steroids the presentation can be devastating, and can progress to ocular infection and even involve the central nervous system. ${ }^{36}$ In addition to the common findings in scleritis of ocular pain, redness, and blurred vision, patients with Nocardia-related scleritis have nodular lesions that present as pointed abscesses. ${ }^{34}$ Nocardia should be suspected when scleral inflammation does not respond to intensive first-line antibiotic therapy. ${ }^{36}$ Direct smear is usually unrevealing; diagnosis of Nocardia is more easily made with Gram stain or acid-fast stain. ${ }^{34}$ One case of Nocardia scleritis showed full resolution with debridement and systemic trimethoprim/ sulfamethoxazole, ${ }^{37}$ but it has also been shown to have very good sensitivity to amikacin. ${ }^{36}$

Other rare fungi have been documented to cause scleritis. Pseudallescheria boydii, a saprophytic fungus frequently isolated from agricultural soil and polluted water, has been reported to present with extensive involvement of the posterior sclera with abscesses. ${ }^{38}$ Paecilomyces lilacinus is an environmental mold found in soil and vegetation that rarely causes human infection. Chung et al discussed a case of P. lilacinus that presented as multiple scleral abscesses with fibrinoid anterior chamber reaction that then progressed to involve the cornea despite topical and oral antifungals. Debridement and intracameral injection of amphotericin B was required for resolution 4 months later, although it resulted in phthisis and light perception vision. ${ }^{39}$ Lastly, Scedosporium prolificans, an emerging opportunistic fungus, was found to cause late-onset scleritis with a median presentation of 7.6 years after pterygium excision. Jhanji et al reported that voriconazole is the most effective medical therapy for $S$. prolificans scleritis, although it only allowed resolution in six of nine cases. ${ }^{40}$ Thus, it is evident that overall visual prognosis in fungal scleritis is generally poor, possibly because of delayed diagnosis, poor penetration of antifungals into avascular sclera, nonavailability of fungicidal agents, or the ability of organisms to persist in avascular scleral tissue for long periods of time without inciting inflammatory response, allowing progressive worsening. ${ }^{1}$

\section{Treatment}

It has been suggested repeatedly that prevention of infectious scleritis is most important for patient outcome. Avoiding overuse of cautery and adjunctive therapy during surgical procedures may spare the episcleral blood flow and allow better wound healing and resistance to infection. ${ }^{21}$ Most studies advocate the avoidance of bare sclera techniques that leave the ocular surface vulnerable to infection. However, in one study by Tittler et al the use of amniotic membrane over an area of debridement was discouraged, suggesting that it provides maximum exposure for topical antibiotics and prevents the incubated microbes from staying at the site, with adequate re-epithelialization occurring shortly after debridement. ${ }^{41}$ If a bare sclera technique is chosen, close follow-up should be undertaken with examination for scleral ischemia, the first sign of which should prompt conjunctival autografting or other scleral reinforcement. ${ }^{21}$

One large study of patients with infectious scleritis by Hodson et al employed various antimicrobial treatment regimens: $95 \%$ topical, $77 \%$ oral, and $11 \%$ intravitreal therapy. Despite a mean treatment duration of 50 days, medical therapy was adequate as the sole treatment in only $18 \%$ of patients, with most requiring surgical debridement. ${ }^{2}$ A higher rate of enucleation or evisceration was present in those treated solely with medical methods. ${ }^{42}$ Cryotherapy, lamellar or penetrating corneoscleral grafts, or removal of hardware in addition to intensive antibiotics improves overall outcomes in patients. ${ }^{2,43}$ Early and repetitive surgical debridement is heavily advocated in multiple studies. ${ }^{1,13,22,41,42}$ Tittler et al showed a $100 \%$ globe preservation rate, best corrected vision of 20/120 to 20/400, fewer complications, and shorter hospital stays with prompt surgical debridement at diagnosis (within 2.5 days). ${ }^{41}$ Most significantly, it has been shown in multiple studies that the inflamed area is often found intraoperatively to be much larger than clinically judged by slit lamp exam (Figure 6). 1,13,21,22 Furthermore, there is sometimes a notable tunnel lesion of scleral ulcer and small pockets of abscess within necrotic sclera that require very careful exploration. Some patients require a scleral patch graft due to the large area of resulting debridement. ${ }^{1,13}$

The use of a fascia lata grafts in combination with an amniotic membrane graft after surgical debridement for infectious scleritis was used successfully by Zheng et al in two patients, one with Pseudomonas and one with fungal scleritis. Fascia lata was thought to be ideal because of its bioadaptability in size and thickness as well as its good cosmetic appearance. Prior to grafting, necrotic scleral tissue was removed and the surface was irrigated with ceftazidime. The fascia lata was then dissected from the biceps femoris in the leg and placed over the defect with an overlying amniotic membrane or pedicle conjunctival flap, with subconjunctival injection of 

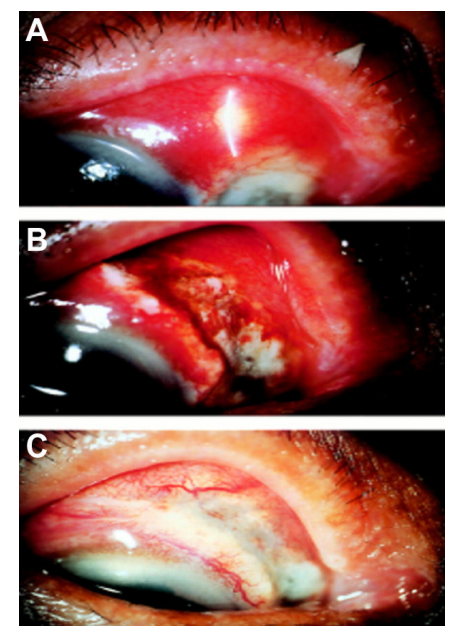

Figure 6 Scleral ulcer before and after debridement

Notes: (A) The scleral ulceration located at the 9 o'clock position. Severe congestion and subconjunctival abscess were noted at 10 o'clock position under the biomicroscope. (B) After the surgical debridement, the scleral wound was left open. in comparison with (A), the involved area was more extensive. (C) Ten days later, the debrided area was healing. (C) 1997 BMJ Publishing Group. Reproduced with permission from: Lin CP, Shih MH, Tsai MC. Clinical experiences of infectious scleral ulceration: a complication of pterygium operation. Br J Ophthalmol. 1997;8I(II):980-983. ${ }^{22}$

ceftazidime followed by oral cefdinir for 4 days. This was the only group to report the use of both a fascia lata and amniotic membrane grafts, and both patients showed evidence of resolution. $^{44}$

Not unlike fascia lata grafts, tectonic scleral reinforcement with preserved pericardium and donor corneal tissue was used successfully in a patient with nocardial scleritis that progressed to scleral perforation and uveal prolapse. This technique along with topical and oral antibiotics allowed salvaging of the eye and a final Snellen visual acuity of $20 / 70 .{ }^{36}$

A variety of medical modalities have been suggested as good adjunctive treatments to surgical debridement. These include systemic and topical antibiotics, subconjunctival injections of antibiotic at both ends of the scleral lesion, and wound irrigation with antibiotic solution one to two times a day followed by normal saline after improvement. ${ }^{22}$ Intraocular antibiotics should be used in all cases of endophthalmitis, and topical steroids should always be initiated only when antibiotics have been reliably used for several days. ${ }^{13}$

Finally, Meallet proposed the use of continuous subpalpebral lavage antibiotics for the treatment of infectious scleritis, reporting resolution of all six cases of scleritis in this study after this treatment. ${ }^{12}$ However, this technique was complicated by hyphema and cataract formation as well as corneal thinning or dense corneal scar requiring corneal transplants, indicating that important adjustments have yet to be made to this technique. Additionally, the question of systemic absorption of this continuous antibiotic regimen was not adequately addressed. ${ }^{12}$

\section{Outcomes}

As may be expected after the discussion in this review, infectious scleritis has worse visual acuity after remission and longer resolution time as compared to noninfectious scleritis. ${ }^{15}$ Not surprisingly, isolated infectious scleritis has better prognosis than sclerokeratitis. Hodson et al reported that $50 \%$ of eyes in their study with infectious scleritis lost functional vision, indicating best corrected Snellen visual acuity less than $20 / 200 .^{2}$

Poor prognostic indicators in infectious scleritis include a presenting vision worse than 20/200 or concomitant keratitis or endophthalmitis, which tend to require enucleation or evisceration more commonly. ${ }^{2}$ Additionally, fungal etiologies tend to have worse prognosis, likely secondary to delays in diagnosis. ${ }^{15}$ However, there is no difference in duration of treatment or need for surgical debridement when comparing bacterial versus fungal cases. ${ }^{2}$ The degree of final vision loss does not significantly correlate with any specific inciting factor, infectious organism, nor the amount of time from symptom initiation to diagnosis. Rather, visual outcome only significantly correlates with low presenting vision. ${ }^{2}$

Hodson et al report that the median time between commencing treatment and resolution of scleritis was 46 days with $50 \%$ of eyes losing functional vision. ${ }^{2}$ Only one case of recurrence was noted after cessation of treatment in this study after apparent full resolution. ${ }^{2}$ Though recurrences are noted commonly as new nodules or necrotic areas during active infectious scleritis, the appearance of such recurrences after achieving full resolution of scleritis is generally rare. ${ }^{2,41}$

Surgical debridement repeatedly resulted in much greater overall improvement in outcome. Sahu et al showed an improvement in vision of greater than two lines in $60 \%$ of patients with use of concurrent debridements as needed. ${ }^{13}$ Reversible causes of vision loss include cataract, fibrotic pupillary membranes, repairable retinal detachments, and corneal opacities. Irreversible causes include chronic retinal and choroidal detachments, glaucoma, or the need for enucleation or evisceration. ${ }^{2,13}$

\section{Conclusion}

Infectious scleritis, though rare, is an important and imminently vision-threatening cause of scleritis. Therefore, it is critical for eye care professionals to maintain infectious etiologies in their differential diagnosis for all patients with presenting symptoms of scleral hyperemia and significant ocular pain. Of particular interest are patients with 
a history of ocular surgery or trauma, regardless of how remote the event, as infections appear to remain dormant within the sclera for months to years before reactivation. In addition, patients' refractory to treatment for presumed autoimmune scleritis should be promptly considered for infectious etiologies, as their immunosuppressive therapy will inevitably worsen their infection. Scleral abscesses and necrosis are common initial signs in infectious scleritis, though many patients will present with more advanced sequelae such as retinal or choroidal detachments and glaucoma. Bacterial, viral, and fungal etiologies exist for infectious scleritis, with Pseudomonas aeruginosa being the most common overall cause and fungi having the worst overall prognosis. Appropriate diagnosis generally requires scleral scrapings with thorough culturing and immunohistochemical staining. However, empiric treatment may be necessary when these laboratory tests return inconclusive. The characteristics of presentation for specific microbes discussed in this review, along with ancillary tests such as ultrasound and optical coherence tomography, may facilitate appropriate diagnosis and therapy in these cases. Effective treatment of infectious scleritis requires both aggressive medical and surgical methods. In addition to microbe-specific medical therapy, repeat drainage and debridement of scleral abscesses and necrotic tissue with the use of various tissue grafts for scleral reinforcement is necessary for resolution. Interestingly, the area of involved sclera is consistently larger intraoperatively than is initially judged on clinical exam, further supporting the importance of surgical exploration. About half of all patients with infectious scleritis maintain functional vision after resolution. Recurrences rarely occur, but highlight the importance of continued close observation for an extended period of time even after clinical resolution in patients with infectious scleritis. With proactive prevention, efficient diagnosis, prompt initiation of aggressive treatment, and close observation, patients with infectious scleritis may have better outcomes.

\section{Disclosure}

The authors report no conflicts of interest in this work.

\section{References}

1. Jain V, Garg P, Sharma S. Microbial scleritis-experience from a developing country. Eye (Lond). 2009;23(2):255-261.

2. Hodson KL, Galor A, Karp CL, et al. Epidemiology and visual outcomes in patients with infectious scleritis. Cornea. 2013;32(4):466-472.

3. Rich RM, Smiddy WE, Davis JL. Infectious scleritis after retinal surgery. Am J Ophthalmol. 2008;145(4):695-699.
4. Cunningham MA, Alexander JK, Matoba AY, Jones DB, Wilhemus KR. Management and outcome of microbial anterior scleritis. Cornea. 2011;30(9):1020-1023.

5. Paula JS, Simão ML, Rocha EM, Romão E, Velasco Cruz AA. Atypical pneumococcal scleritis after pterygium excision: case report and literature review. Cornea. 2006;25(1):115-117.

6. Maskin SL. Infectious scleritis after a diabetic foot ulcer. Am J Ophthalmol. 1993;115(2):254-255.

7. Hwang YS, Chen YF, Lai CC, Chen HS, Hsiao CH. Infectious scleritis after use of immunomodulators. Arch Ophthalmol. 2002;120(8): 1093-1094.

8. Moreno Honrado M, del Campo Z, Buil JA. A case of necrotizing scleritis resulting from Pseudomonas aeruginosa. Cornea. 2009;28(9): $1065-1066$.

9. Meyer JJ, Espandar L, Marx DP, Vitale A, Moshirfar M. Pseudomonas scleritis resistant to fourth-generation fluoroquinolones in a patient without prior trauma or surgery. Ocul Immunol Inflamm. 2008;16(3): $127-129$.

10. Su CY, Tsai JJ, Chang YC, Lin CP. Immunologic and clinical manifestations of infectious scleritis after pterygium excision. Cornea. 2006;25(6):663-666.

11. Altman AJ, Cohen EJ, Berger ST, Mondino BJ. Scleritis and Streptococcus pneumoniae. Cornea. 1991;10(4):341-345.

12. Meallet MA. Subpalpebral lavage antibiotic treatment for severe infectious scleritis and keratitis. Cornea. 2006;25(2):159-163.

13. Kumar Sahu S, Das S, Sharma S, Sahu K. Clinico-microbiological profile and treatment outcome of infectious scleritis: experience from a tertiary eye care center of India. Int J Inflam. 2012;2012:753560.

14. Bhat PV, Jakobiec FA, Kurbanyan K, Zhao T, Foster CS. Chronic herpes simplex scleritis: characterization of 9 cases of an underrecognized clinical entity. Am J Ophthalmol. 2009;148(5):779-789. e2.

15. Ahn SJ, Oh JY, Kim MK, Lee JH, Wee WR. Clinical features, predisposing factors, and treatment outcomes of scleritis in the Korean population. Korean J Ophthalmol. 2010;24(6):331-335.

16. Margo CE, Pavan PR. Mycobacterium chelonae conjunctivitis and scleritis following vitrectomy. Arch Ophthalmol. 2000;118(8): $1125-1128$.

17. Lyne AJ, Lloyd-Jones D. Necrotizing scleritis after ocular surgery. Trans Ophthalmol Soc U K. 1979;99(1):146-149.

18. Gharaee H, Khalife M, Poor SS, Abrishami M. Infectious scleritis after subtenon triamcinolone acetonide injection. Ocul Immunol Inflamm. 2011;19(4):284-285.

19. Seth RK, Gaudio PA. Nocardia asteroides necrotizing scleritis associated with subtenon triamcinolone acetonide injection. Ocul Immunol Inflamm. 2008;16(4):139-140.

20. Gonzalez-Gonzalez LA, Molina-Prat N, Doctor P, Tauber J, Sainz de la Maza MT, Foster CS. Clinical features and presentation of infectious scleritis from herpes viruses: a report of 35 cases. Ophthalmology. 2012;119(7):1460-1464.

21. Hsiao CH, Chen JJ, Huang SC, Ma HK, Chen PY, Tsai RJ. Intrascleral dissemination of infectious scleritis following pterygium excision. Br J Ophthalmol. 1998;82(1):29-34.

22. Lin CP, Shih MH, Tsai MC. Clinical experiences of infectious scleral ulceration: a complication of pterygium operation. Br J Ophthalmol. 1997;81(11):980-983.

23. Riono WP, Hidayat AA, Rao NA. Scleritis: a clinicopathologic study of 55 cases. Ophthalmology. 1999;106(7):1328-1333.

24. Sainz de la Maza M, Molina N, Gonzalez-Gonzalez LA, Doctor PP, Tauber J, Foster CS. Clinical characteristics of a large cohort of patients with scleritis and episcleritis. Ophthalmology. 2012;119(1): $43-50$.

25. Nguyen P, Yiu SC. Imaging studies in a case of infectious scleritis after pterygium excision. Middle East Afr J Ophthalmol. 2012;19(3): 337-339.

26. Arora R, Shroff D, Narula R, Chauhan D, Mehta DK. Inverse hypopyon as the presenting feature of infectious scleritis in a case of tropical pyomyositis. Can J Ophthalmol. 2006;41(6):769-771. 
27. Lee JE, Oum BS, Choi HY, Lee JS. Methicillin-resistant Staphylococcus aureus sclerokeratitis after pterygium excision. Cornea. 2007;26(6): $744-746$.

28. Lin HC, Ma DH, Chen YF, Yeh LK, Hsiao CH. Late-onset intrascleral dissemination of Stenotrophomonas maltophilia scleritis after pterygium excision. Cornea. 2011;30(6):712-715.

29. Ramos-Esteban JC, Jeng BH. Posttraumatic Stenotrophomonas maltophilia infectious scleritis. Cornea. 2008;27(2):232-235.

30. Kesen MR, Edward DP, Rao NA, Sugar J, Tessler HH, Goldstein DA. Atypical infectious nodular scleritis. Arch Ophthalmol. 2009;127(8):1079-1080.

31. Metta H, Corti M, Brunzini R. Disseminated infection due to Mycobacterium chelonae with scleritis, spondylodiscitis and spinal epidural abscess. Braz J Infect Dis. 2008;12(3):260-262.

32. Poon A, MacLean H, McKelvie P. Recurrent scleritis in lepromatous leprosy. Aust N Z J Ophthalmol. 1998;26(1):51-55.

33. Schuman JS, Weinberg RS, Ferry AP, Guerry RK. Toxoplasmic scleritis. Ophthalmology. 1988;95(10):1399-1403.

34. DeCroos FC, Garg P, Reddy AK, et al; Hyderabad Endophthalmitis Research Group. Optimizing diagnosis and management of nocardia keratitis, scleritis, and endophthalmitis: 11-year microbial and clinical overview. Ophthalmology. 2011;118(6):1193-1200.

35. Fincher T, Fulcher SF. Diagnostic and therapeutic challenge of Aspergillus flavus scleritis. Cornea. 2007;26(5):618-620.

36. Ramos-Esteban JC, Servat JJ, Silva RS, Ambrósio R, Tauber S, Bia F. Necrotizing nocardial scleritis after combined penetrating keratoplasty and phacoemulsification with intraocular lens implantation: a case report and review of the literature. Arq Bras Oftalmol. 2007;70(2):355-359.
37. Maruo H, Shiraishi A, Hara Y, Maruo Y, Ohashi Y. Necrotizing nocardial scleritis successfully treated with surgical debridement and topical polyvinyl alcohol iodine and antibiotics. J Ocul Pharmacol Ther. 2011;27(4):415-418.

38. Taravella MJ, Johnson DW, Petty JG, Keyser RB, Foster CS, Lundberg BE. Infectious posterior scleritis caused by Pseudallescheria boydii. Clinicopathologic findings. Ophthalmology. 1997;104(8):1312-1316.

39. Chung PC, Lin HC, Hwang YS, et al. Paecilomyces lilacinus scleritis with secondary keratitis. Cornea. 2007;26(2):232-234.

40. Jhanji V, Yohendran J, Constantinou M, Sheorey H, Vajpayee RB. Scedosporium scleritis or keratitis or both: case series. Eye Contact Lens. 2009;35(6):312-315.

41. Tittler EH, Nguyen P, Rue KS, et al. Early surgical debridement in the management of infectious scleritis after pterygium excision. J Ophthalmic Inflamm Infect. 2012;2(2):81-87.

42. Reynolds MG, Alfonso E. Treatment of infectious scleritis and keratoscleritis. Am J Ophthalmol. 1991;112(5):543-547.

43. Alfonso E. Surgical intervention in infectious keratoscleritis. Arch Ophthalmol. 1994;112(8):1017-1018.

44. Zheng X, Kodama T, Goto T, Ohashi Y. Autologous fascia lata grafts for scleral repair in eyes with infectious necrotizing scleritis. Arch Ophthalmol. 2011;129(9):1225-1227.

45. Raiji VR, Palestine AG, Parver DL. Scleritis and systemic disease association in a community-based referral practice. Am J Ophthalmol. 2009;148(6):946-950.

46. Watson PG, Hayreh SS. Scleritis and episcleritis. Br J Ophthalmol. 1976;60(3):163-191.
Clinical Ophthalmology

\section{Publish your work in this journal}

Clinical Ophthalmology is an international, peer-reviewed journal covering all subspecialties within ophthalmology. Key topics include: Optometry; Visual science; Pharmacology and drug therapy in eye diseases; Basic Sciences; Primary and Secondary eye care; Patient Safety and Quality of Care Improvements. This journal is indexed on Submit your manuscript here: http://www.dovepress.com/clinical-ophthalmology-journal

\section{Dovepress}

PubMed Central and CAS, and is the official journal of The Society of Clinical Ophthalmology (SCO). The manuscript management system is completely online and includes a very quick and fair peer-review system, which is all easy to use. Visit http://www.dovepress.com/ testimonials.php to read real quotes from published authors. 